\title{
HELIUM GAS EVACUATION IN SUPERCONDUCTING RFQ STRUCTURE
}

\author{
A. Lombardi, G. Bisoffi, F. Chiurlotto, E. Tovo, A.M. Porcellato, L. Badan, \\ INFN-LNL via Romea 4, I35020 Legnaro (PD), Italy, \\ L. Taffarello, INFN Padova, via Marzolo 8, Padova (PD), Italy \\ G. Navarro, M. Antonello, M. Masi \\ Università di Padova, Dipartimento di Ingegneria Meccanica, via Venezia 1, Padova, Italy
}

\begin{abstract}
The PIAVE injector for the Legnaro Accelerator complex is an accelerating machine made of superconducting resonators. One of them, the superconducting RFQ, needs to be cooled by the helium bath on the whole outer surfaces. In particular the region of the electrode tips and the lower vertical electrode are interested to RF power dissipation and can be a trapped volume of liquid. During the operation the liquid evaporates and the produced gas needs to be evacuated, in order to cool the structure properly. The problem of gas production and evacuation from a trapped volume of liquid helium has been studied and solved. Studies of the possible evacuation systems and of the experimental apparatus are presented.
\end{abstract}

\section{Scope of the test}

The superconducting RFQ (Radio Frequency Quadrupole) structures [1], made of niobium sheets formed and eb-welded, are cooled by the direct contact with the liquid helium bath. Figure 1 shows the longitudinal section of the test cryostat. The outer tank, the thermal shield operated at liquid nitrogen, the liquid helium reservoir and the SRFQ vertical electrodes can be seen.

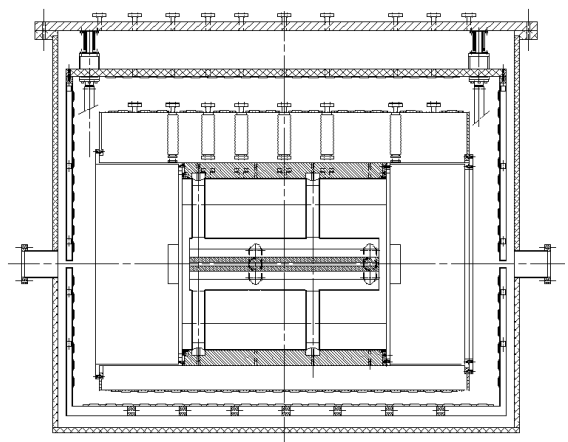

Figure 1: The SRFQ structure inside the test cryostat.

The RF power on the wall of the resonator is dissipated inside the liquid helium bath through the evaporation of the liquid with a gas production rate of: $1 \mathrm{~W} \Leftrightarrow 1 . \mathrm{m}^{3} / \mathrm{h}$.

The SRFQ electrodes are hollowed and they are filled by liquid helium. The geometrical disposition of the vertical electrodes allows the trapping of the helium gas evaporated by the RF power dissipation on the bottom electrode.

The scope of the experiment is to verify if the natural draining due to the hydrostatic pressure of the liquid helium itself through a siphon ending over the liquid helium level and controlled by a needle valve is sufficient to keep the trapped volume filled by liquid. We want to avoid the use of a forced flow of liquid helium because of the sensitivity of the resonator to the mechanical noise.

The experiment was performed using a model of the trapped volume made with an upside-down cylinder isolated by the liquid helium. The thermal insulation of the apparatus is made with a chamber around the cylinder simulating the electrode (fig. 2). The inter-space is evacuated to simulate the inner part of the SRFQ resonator. The RF power was simulated by means of a resistor placed inside the trapped volume.

\section{Theoretical description}

The possibility of draining the gas helium from the SRFQ electrode is subject to the value of the liquid height above the trapped volume and to the pressure heads of the draining system.

The draining is realised via a standard tube of some $\mathrm{mm}$ diameter going from the lower electrode to the top of the liquid helium reservoir. The tube will run outside the liquid helium reservoir to avoid condensation of the cold vapour.

The present analysis is devoted to estimate the pressure heads of the elements of the experimental apparatus (figure 2) so as to verify the pressure drop of the needle valve which is the only home made element.

The methodology, indicated for standard pipes [2, 3], can be used to calculate the pressure losses into pipes with small diameter, considering a relative roughness large than in the normal pipe, because there is a scale effect. The evaporation rate for the liquid helium in standard condition is $1.4 \mathrm{l} / \mathrm{h}$ per dissipated watt.

During the standard operation the RF power dissipation foreseen for the SRFQ, in the area interested by the gas trapping problem, is of the order of $2 \mathrm{~W}$ and exceptionally it produces a rate flow equivalent to $4 \mathrm{~W}$.

The calculation presented here are related to the experiment where the maximum power dissipated inside the test volume was $2.5 \mathrm{~W}$

The pressure heads are defined as localised pressure drops and distributed ones.

The localised losses, $h_{i}$, are calculated at the mouthpiece of the pipe, at the bow elements, at the section contraction of the pipe, at the outlet of the pipe and at the valve, whereas the distributed ones are defined in relation with the length and the diameter of the pipe. 
The pressure losses due to the inlet sections as well as for the element contraction are calculated by the following equation:

$$
\mathrm{h}_{\mathrm{i}}=\mathrm{Q}_{\mathrm{g}} / \mathrm{C}_{\mathrm{i}}\left(\mathrm{K}_{\mathrm{c}}\right)
$$

with $Q_{g}$ the volumetric rate flow and $\mathrm{C}_{\mathrm{i}}$ the loss coefficient for the localised losses described in reference [4]. Using figure 2 in the ref. [4] we fixed the loss coefficient $\mathrm{K}_{\mathrm{c}}$ equal to 0.622 for the inlet to the $12 \mathrm{~mm}$ pipe and 0.605 for the transition to the $3 \mathrm{~mm}$ pipe. For the successive transition from $3 \mathrm{~mm}$ to $2 \mathrm{~mm}$ pipe section we used $\mathrm{K}_{\mathrm{c}}=0.42$.

An equation similar to (1) is used for the computation of the pressure drop in the bow elements. The diagram of figure 3 in [5] defines the loss coefficient $K_{b}$ as a function of the bending angle of the bow element. In the reference the value of the Reynolds number $(R e)$ is fixed equal to $10^{6}$ and in this calculation it has been used a correction factor when the value of $R e$ is different. The pressure drop at the outlet section of the pipe is still calculated using an equation similar to (1), fixing equal to 1 the value of the loss coefficient.

The distributed losses in the pipe are defined by the following equation:

$$
h_{d}=f \cdot \frac{L}{D_{b}} \cdot \frac{8 \cdot Q_{g}^{2}}{g \cdot \pi^{2} \cdot D_{b}^{4}}
$$

with $\mathrm{L}$ is the tube length, $\mathrm{D}_{\mathrm{b}}$ the tube diameter and the friction factor $f$ is calculated using the Hagen-Poiseuille law for laminar flow.

In the turbulent flow Jain's law is applicable for Reynolds number less than $10^{8}$ and $f$ follows the relation:

$$
f=\frac{0.25}{\left[\log _{10}\left(\frac{5.74}{\operatorname{Re}^{0.9}}+\frac{e}{3.7}\right)\right]^{2}}
$$

The friction loss is defined in relation to the equivalent relative roughness $e$.

In the experimental apparatus we decided to have two different tube diameters in two sections, 3 and $2 \mathrm{~mm}$ respectively. This is done so as to operate the needle valve in a convenient opening range for all the operation conditions. The pipe length $L$ is the sum of all the pipe straight sections.

If $\Delta p_{a}$ is the total pressure drop, it is possible to compute the pressure drop $\Delta p_{v}$ that the valve causes to obtain the equilibrium with the static head $z_{s t}$ of liquid helium.

$\Delta p_{v}=\rho_{l} \cdot g \cdot z_{s t}-\rho_{g} \cdot g \cdot\left(z_{E}-z_{B}\right)-\Delta p_{a}$

Here $\left(z_{E}-z_{B}\right)$ is the elevation difference of the gas helium between the inlet section of the pipe (B) and the position of the valve (E), $\rho_{l}$ and $\rho_{g}$ are the liquid and gas densities respectively.

Defined the valve type, it is possible to plot the parametric curve to define the loss coefficient for various fully and partially open valve. Therefore it is possible to obtain also a working range for the values of pressure head in the valve in relation with the gas flow rate when the valve opening passes from fully open to closed. The values of the physical quantities of the helium and the results of the numerical simulation of the pressure head are shown in table 1 . The reasonable valve pressure drop shows that the pipe diameters with 2 and $3 \mathrm{~mm}$ are suitable to obtain the draining of the small gas helium rates from the trapped volume.

\begin{tabular}{|c|c|c|c|}
\hline $\begin{array}{c}\text { Dissipated } \\
\text { power } \\
(\mathrm{W})\end{array}$ & $\begin{array}{c}\text { Gas flow rate } \\
\left(\mathrm{mm}^{3} / \mathrm{s}\right)\end{array}$ & $\begin{array}{c}\Delta \mathrm{p}_{\mathrm{a}} \\
(\mathrm{Pa})\end{array}$ & $\begin{array}{c}\Delta \mathrm{p}_{\mathrm{v}} \\
(\mathrm{Pa})\end{array}$ \\
\hline 0.1 & 292 & 1.2 & 536.2 \\
\hline 0.54 & 1576 & 26.3 & 488.5 \\
\hline 1.1 & 3210 & 104.7 & 394.7 \\
\hline 1.5 & 4377 & 190.4 & 273.4 \\
\hline 2.1 & 6127 & 367.6 & 37.4 \\
\hline 2.3 & 6711 & 438.6 & 188.3 \\
\hline 2.5 & 7295 & 515.8 & 124.6 \\
\hline$\rho_{\mathrm{l}}=125 \mathrm{~kg} / \mathrm{m}^{3}$ & $\rho_{\mathrm{g}}=17 \mathrm{~kg} / \mathrm{m}^{3}$ \\
\hline$\mu_{\mathrm{g}}=1.3 * 10^{-6} \mathrm{~kg} /(\mathrm{m} \cdot \mathrm{s})$ & $\mathrm{e}=0.0075$ \\
\hline $\mathrm{z}_{\mathrm{E}}-\mathrm{z}_{\mathrm{B}}=500 \mathrm{~mm}$ & $\begin{array}{l}\mathrm{z}_{\mathrm{st}}=375 \mathrm{~mm}+\text { liquid helium } \\
\text { hydrostatic height }\end{array}$ \\
\hline
\end{tabular}

Table 1. Theoretical estimations of the needle valve pressure heads.

We can cope with the eventual dissipation of a higher $\mathrm{RF}$ power using a $4 \mathrm{~mm}$ draining pipe.

\section{The experimental apparatus}

Before using the siphon draining system inside a complex structure such as the SRFQ resonators test cryostat, which needs long cooling down and warm up periods, we decided to construct a model (figure 2).

It consists in an upside down cylinder included into a vacuum jacket and suspended to the top flange of a dewar.

In figure 2 one can see: the top flange; the radiation shields; the liquid helium level meter; the gas draining system and the needle valve.

Inside the trapped volume there are four temperature sensors, one resistor for the power dissipation and a liquid helium level meter.

The draining tube, which ends with the valve above the liquid level, is thermally isolated by the liquid helium bath being inside a vacuum jacket.

In order to be able to warm up the liquid which might fill up the draining tube, a second resistor is placed inside the vacuum jacket (particular number 4 ).

The experiments consisted in powering the resistor inside the trapped volume with a known current and finding the proper opening of the needle valve that reaches the equilibrium between the gas production and the gas draining.

The experiments were done with different liquid helium level above the inlet of the draining tube. This was done to simulate the different operational conditions of the real resonator inside its cryostat. The various liquid heights are in table 2. 
The liquid helium level inside the trapped volume was measured using the properly calibrated temperature sensors and a level meter.

The aim of the tests was to check if the system is able to deal with the power dissipation of the order of $2.5 \mathrm{~W}$, which is the maximum power foreseen for the lower electrode of the SRFQ (figure 1).

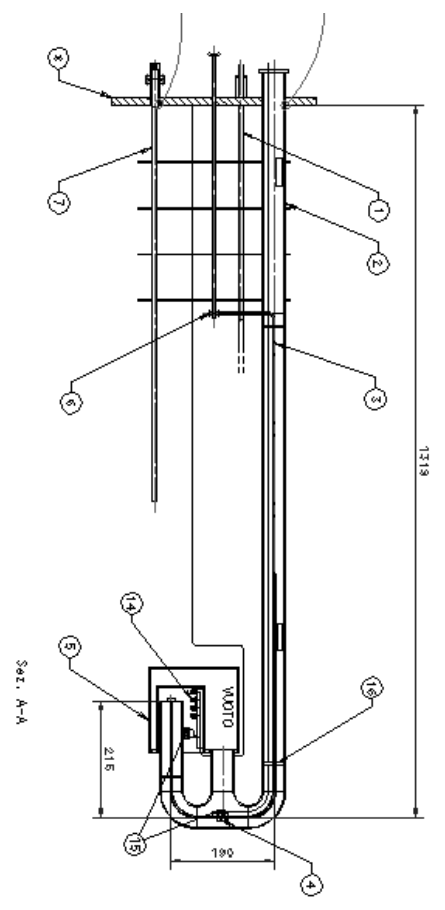

Figure 2: The experimental apparatus.

The experimental procedure adopted for the tests was to insert the apparatus inside a dewar and cool it down to liquid helium temperature. Once the trapped volume was filled with liquid helium the needle valve was closed and the resistor was powered with a known input power. The gas filled the volume and the liquid level underneath the upside-down cylinder was controlled with the level meter and with calibrated temperature sensors.

The second operation was to open the needle valve to a fix opening and see if the gas was drained properly and in a stable flux in equilibrium with the gas production due to the power dissipation.

During the tests the liquid level inside the whole dewar was controlled with a second level meter in order to check the hydrostatic pressure to compare with the calculation.

The open questions were:

Does we need a pumping system to drain the gas or is the hydrostatic pressure of the liquid helium enough? Is the size of the draining tube correct? Can the draining system be recovered if some liquid flows inside the draining tube, avoiding bubbling and mechanical noise? Are the theoretical assumptions on the pressure losses correct?

\section{Experimental results}

The experimental results are summarised in table 2 .
The draining system worked properly and the draining tube did not show a severe problem with its filling up with liquid helium. In effect the tube was not filled with liquid even when the liquid helium level overcome the tube inlet. This is connected with the level of the insulation vacuum. During the first test the vacuum was in the $10^{-4}$ mbar range and we did not have problem with liquid into the tube; during the successive tests the vacuum deteriorated and we had to power the second resistor to evaporate the liquid inside the draining tube. Once the liquid is removed the system worked properly again.

\begin{tabular}{|c|c|c|}
\hline $\begin{array}{c}\text { Dissipated } \\
\text { Power } \\
(\mathrm{W})\end{array}$ & $\begin{array}{c}\text { Valve opening } \\
\text { at equilibrium } \\
(\%)\end{array}$ & $\begin{array}{c}\text { Liquid helium } \\
(\mathrm{mm})\end{array}$ \\
\hline .1 & 18.5 & 159 \\
\hline .54 & 18.5 & 141 \\
\hline 1.1 & 27.1 & 128 \\
\hline 1.5 & 47 & 99 \\
\hline 2.1 & 100 & 51 \\
\hline 2.3 & 100 & 232 \\
\hline 2.5 & No equil. & 243 \\
\hline 2.1 & 77 & 328 \\
\hline
\end{tabular}

Table 2: Experimental results.

\section{Conclusions}

The experimental results show that it is possible to drain the trapped gas produced in an upside-down container using the hydrostatic pressure.

We will insert the system inside the SRFQ resonator using, for this application up to few watts of dissipation, a $3 \mathrm{~mm}$ diameter tube and a proper needle valve operated at room temperature.

The thermal load to keep the draining tube empty will be provided by the irradiation to the liquid nitrogen shield because the tube will run outside the liquid helium reservoir.

\section{Acknowledgements}

We acknowledge very much the mechanical support given by Enzo Bissiato concerning the realisation of the needle valve.

\section{References}

[1] A. Pisent et al. The new LNL injector PIAVE, based on a superconducting $R F Q$ Proc. of the European Particle Accelerator Conf., Stockholm, Sweden, 1998, Institute of Physics Publishing Bristol and Philadelphia, pp758-760 1998

[2] Schlichting, H., Boundary-Layer Theory, McGraw-Hill, Seventh Edition, New York, 1979.

[3] Ghetti, A., Idraulica, Libreria Cortina, Second Edition, Padova, 1991.

[4] Haghighi, K., Bralts, V.F., and Segerling, L.J., "Finite Element Formulation of Tee and Bend Components in Hydraulic Pipe Network Analysis", Transactions of the ASAE 31(6): 1750-1758, 1988.

[5] Haghighi, K., Bralts, V.F., Mohtar, R., and Segerling, L.J., "Modeling Expansion/Contraction, Valve and Booster Pump in Hydraulic Pipe Network Analysis: A Finite Element Approach", Transactions of the ASAE 32(6): 1945-1953, 1989. 